\title{
Criminología y política criminal: la necesidad de un foro de discusión
}

\author{
"Facts are subversive”, IF Stone
}

\author{
Dr. Juanjo Medina iD \\ Profesor Titular de Criminología y Política Social \\ Departamento de Ciencia Social Aplicada, Universidad de Manchester
}

\section{Delincuencia y política criminal}

La delincuencia y la respuesta social e institucional a la misma se han convertido en uno de los temas más destacados en los medios de comunicación españoles. Las principales formaciones políticas españolas también han otorgado una mayor relevancia a estas cuestiones en sus discursos y pronunciamientos públicos (Medina, En Prensa). Sin embargo, a pesar de la relevancia que se da a estos temas es también evidente la ausencia de un debate serio y riguroso sobre estas cuestiones. En el ámbito político criminal López Rey (1985) hace ya bastantes años decía que en España se hacía política criminal "de sillón" y en los albores del nuevo milenio las cosas no parecen haber cambiado mucho a pesar de la estabilización del modelo constitucional y democrático.

En España existe la sensación de que las diferentes formaciones políticas que han tenido la oportunidad de gobernar y de cambiar la situación se han limitado a cambiar la legislación. Un marco legislativo adecuado es evidentemente necesario pero, por un lado, las leyes no se implementan por sí solas, ni siempre tienen el efecto esperado y, por otra parte, el juicio sobre lo que constituye un marco legislativo adecuado es algo que en todo caso ha de ser evaluado conforme a los criterios de justicia y eficacia (Hassemer y Munoz Conde, 1989). Sobre lo primero nuestros compañeros los penalistas han generado una literatura someramente amplia. Pero la justicia de una norma depende en gran medida de cómo se aplica y a quién se aplica. Y sobre estas cuestiones, y sobre las cuestiones de eficacia, nuestro conocimiento en España es muy limitado por la falta de suficientes estudios criminológicos.

En España este tipo de consideraciones sobre eficacia desgraciadamente suele escapar las discusiones políticas sobre la delincuencia y la justicia penal. El resultado es que se aprueban leyes sin que existan los medios para implementarlas, se definen como problemas sociales situaciones cuya seriedad y complejidad se desconoce, se legisla sobre situaciones cuya naturaleza no se comprende y una vez se aprueban las leyes nunca se piensa en evaluar en qué medida cumplen su cometido. En España la discusión sobre políticas públicas e iniciativas legislativas en el ámbito político criminal parece producirse al margen de un análisis sosegado de datos objetivos sobre necesidades sociales, adecuación de la solución al problema, necesidades de la implementación y posibles efectos (Diez Ripollez y Cerezo,2001). Algo que no debería extrañarnos demasiado si nos paramos a reflexionar sobre el deficiente grado de inversión en $\mathrm{I}+\mathrm{D}$ en España. 
Uno de los correlatos de ello es la creciente presencia de discursos populistas tendentes a reivindicar lo que el criminólogo británico David Garland (2001) ha definido como una "penalidad expresiva" tendente a demandar mano dura o lo que nuestros penalistas denominan "un derecho penal del enemigo" (Silva Sánchez, 2001), así como la proliferación de mitos infundados en las discusiones sobre estas cuestiones. Otra de las consecuencias es que nuestras soluciones al problema de la inseguridad ciudadana no son tan correctas, desde el punto de vista de justicia y eficacia, como podrían ser y que incluso cuando lo son no tenemos los dispositivos adecuados para saberlo.

Los debates actuales sobre inseguridad ciudadana son un buen ejemplo de cómo se están ignorando los escasos datos existentes sobre este fenómeno en nuestro país. Si uno se limita a valorar el problema de la inseguridad ciudadana desde la perspectiva de los medios de comunicación social y las principales formaciones políticas españolas, uno debería llegar a la conclusión de que las cosas han empeorado de forma considerable en tiempos recientes y que el nivel de miedo al delito en España ha aumentado en los últimos años. Sin embargo, los únicos datos fiables sobre sentimientos subjetivos de inseguridad ciudadana en nuestro país, como veremos a continuación, reflejan una situación muy diferente.

Las autoridades españolas, por lo general, no realizan investigación criminológica de calidad similar a la existente en otros países, pero la Comisión Europea ha conseguido lo que las autoridades nacionales no han logrado: producir datos fiables y comparables sobre los sentimientos de inseguridad ciudadana en nuestro país. El CIS suele incluir preguntas sobre estas cuestiones, pero empleando generalmente medidas inadecuadas, que cambian con frecuencia y empleando encuestas que varían en sus diseños muestrales lo que complica la producción de series temporales. Tres Euro-barómetros, el primero realizado en 1996, seguido de uno en el año 2000 y otro en el 2002 han generado medidas más fiables y consistentes que nos permiten evaluar en qué medida los ciudadanos españoles se sienten más preocupados hoy por cuestiones de inseguridad ciudadana que en $1996^{1}$.

Las conclusiones que se derivan de un análisis sosegado y detallado de los Eurobarómetros es que los sentimientos de inseguridad ciudadana en nuestro país, por lo demás similares a la media europea, no han cambiado en este periodo. La percepción del problema de drogas, de hecho, ha disminuido acercándonos a la media europea por encima de la que nos encontrábamos en 1996. Cuando se pregunta a los ciudadanos en qué medida piensan que serán víctimas de delitos en el curso del 2003, las cifras que se compilan ubican a España con niveles similares a la media de la Unión Europea con la excepción del riesgo de atracos a pisos. Solamente el 18\% de los españoles piensan que van a sufrir este delito en comparación con el $26 \%$ de los ciudadanos de la Unión Europea.

Es difícil contrastar estas cifras sobre sentimientos subjetivos de inseguridad ciudadana con datos objetivos sobre el volumen de delitos registrados por las fuerzas y cuerpos de seguridad del Estado, dado el hermetismo del Ministerio de Interior, o con 
datos basados en encuestas de victimación, dado que las encuestas españolas al respecto son ejemplos sobre como no conducir encuestas de victimación (el Ministerio de Interior se empeña en diseñar cuestionarios sui generis en lugar de participar en la Encuesta Internacional del Delito, un proyecto respaldado por 17 naciones industrializadas, incluyendo Cataluña, y otros países en vía de desarrollo). Mientras que en Inglaterra y Gales, por poner un ejemplo, las estadísticas policiales se publican de forma cuatrimestral, en nuestro país los datos más recientes -recogidos mensualmente por cierto- que se pueden obtener vía la publicación restringida del Anuario Estadístico del Ministerio de Interior o en la página web del Ministerio tienen más de dos años de antigüedad y las publicaciones del Sindicato Unificado de Policía son demasiado oscuras, metodológicamente controvertidas ${ }^{\mathrm{ii}} \mathrm{e}$ interesadas como para ser aceptadas sin matices.

En todo caso, parece evidente, si uno ha de creer los Eurobarómetros, que en España no se trata de que la ciudadanía de pronto se sienta particularmente atemorizada por estas cuestiones. Los sentimientos de inseguridad ciudadana no han aumentado en nuestro país, incluso, lo que es más sorprendente, a pesar de los numerosos discursos públicos que reflejan y promueven esta idea. Si a esto añadimos uno de los datos más preocupantes del Euro-barómetro del 2002, que los españoles se encuentran por encima de la media europea en su creencia de que penas más duras son necesarias, en el contexto de una población carcelaria bastante elevada, es evidente que lo último que necesitamos por parte de la clase política son discursos alarmistas tendentes a simplificar el problema y a fomentar el desarrollo de una punitividad inconsecuente con las aspiraciones de un Estado social y democrático de derecho.

La forma en que estos datos se ignoran es consistente con la hipótesis de que el modelo de la política criminal "de sillón” criticado por Lopez Rey (1985) ha sido superado en el siglo XXI por una democracia política que opera en un mundo mediático de realidad virtual, en el que la apariencia es más importante que la realidad. Sin embargo, frente a un modelo de hacer política criminal como éste existen formulas que prestan una mayor atención a la realidad social sobre la que se quiere incidir. Por ejemplo, en la década de los 60, los presidentes estadounidenses Kennedy, Johnson y Nixon dedicaron una atención especial a cuestiones sociales. El gobierno norteamericano desarrolló una serie de programas sociales orientados a mejorar la situación de los pobres en los Estados Unidos. Estos programas eran, en términos económicos, muy costosos por lo que era preciso saber si los mismos estaban obteniendo los resultados esperados. En Estados Unidos, como en España, la financiación de los programas públicos de tipo social o de tipo penal para atajar la delincuencia son financiados con el dinero de los contribuyentes, con nuestros impuestos, y no cabe duda que el uso de fondos públicos requiere que los mismos sean empleados de forma adecuada y transparente para evitar el despilfarro. Este proceso de evaluación, además de las propias intervenciones, sirven para cimentar la confianza del ciudadano en las instituciones públicas, particularmente en un momento en el que existe un cierto sentimiento de crisis de opinión en torno al funcionamiento de la justicia.

En el contexto estadounidense el sociólogo Donald Campbell, uno de los pilares de la metodología de las ciencias sociales, se dedicó a desarrollar una serie de criterios 
que ayudaran a la evaluación de estas iniciativas. Campbell llegó a la conclusión de que la experimentación social era la mejor forma de obtener conclusiones válidas sobre el éxito de las intervenciones propuestas. Campbell fue más lejos y llego a proponer una modelo "ideal" de desarrollo de políticas basados en la experimentación científica (Campbell, 1988). El mundo de la política es mucho más complicado de lo que este modelo utópico de claras resonancias modernistas admite y la literatura sobre el proceso de confección e implementación de políticas públicas ha dado buena cuenta de ello (Parsons, 1995; National Audit. Office, 2003). Existen numerosas teorías y marcos de explicación en las ciencias políticas que reconocen la complejidad del proceso de definición de problemas sociales, de creación de agendas, aprobación de iniciativas e implementación de las mismas (Parsons, 1995; Hill, 1997). Con todo, no cabe duda de que existe un espacio necesario para la investigación empírica y para la evaluación científica en el contexto de la política criminal. Resulta cuanto menos curioso que en España, donde la importación de soluciones político criminales es evidente si uno compara nuestra legislación con la de otros países de nuestro entorno, no se copien otros elementos políticos como lo es la práctica de la evaluación científica de estas políticas.

Es cierto también que el proceso por el cual estudios científicos llegan a influir la toma de decisiones políticas es complejo y está marcado por una serie de obstáculos importantes (NAO, 2003). En los tiempos que corren sería demasiado ingenuo pensar que la investigación es un proceso puro e invulnerable a influencias sociales, políticas y económicas. Cuantos participamos en la empresa científica también participamos en el mundo social que investigamos y sobre el que queremos incidir. Pero existen dificultades adicionales: el lenguaje científico es oscuro, el ritmo de la investigación científica es mucho más lento que el ritmo de la actividad política, los resultados de cualquier investigación son normalmente complejos e igualmente complejas son las implicaciones prácticas que se pueden derivar de ellas, etc. Finalmente, el uso de "soluciones científicas" a los problemas que nos confrontan en numerosas ocasiones crean tantos problemas como solucionan (Beck, 1992). A pesar de todo ello, sigue siendo más razonable apostar por un modelo de política criminal que toma en consideración un análisis científico y objetivo de los problemas sobre los que se quiere incidir y de las soluciones que se proponen al mismo que apostar por un modelo de política criminal que ignora estas realidades sociales y se basa en lo anecdótico, el llamado "sentido común" y la demagogia.

La investigación científica, por otra parte, también es susceptible de abuso y de manipulación política. "Lies, Damn Lies, and Statistics” reza el conocido dicho sociológico. El experto en encuestas es consciente de cómo pequeñas y sutiles variaciones en la redacción de una pregunta es capaz de generar resultados muy diferentes ${ }^{\text {iii }}$. En un área tan cargada emocionalmente como lo es el estudio de la delincuencia el investigador tiene que ser particularmente consciente de ello. Pero no solo las cifras estadísticas son susceptibles de manipulación más o menos consciente, este es un problema más general de las ciencias sociales. En España, como en cualquier otro país, existen numerosos ejemplos de estudios científicos puestos al servicio del poder para legitimar determinadas soluciones políticas. El Barómetro del CIS de Enero del 2003 es un buen ejemplo de ello. Determinadas formaciones políticas españolas han 
apostado por el recurso a la mano dura como la forma de atajar el problema de la inseguridad ciudadana en nuestro país y en ese contexto desde un organismo público se formula una encuesta que plantea al publico su grado de apoyo a una serie de medidas que no se explican debidamente, que reducen de forma sobresaliente la complejidad del tema, y que no se comparan con otras medidas ${ }^{\text {iv }}$. Todos estamos a favor de que se tomen medidas contra la delincuencia, la cuestión es cuáles son las medidas que preferimos. En la medida en que no se ofrezca al encuestado la posibilidad de elegir entre diferentes medidas, los resultados de este tipo de estudios tienden a ser poco fiables y tienden a ofrecer resultados que favorecen resoluciones más punitivas. Este Barómetro presenta preguntas que dada su redacción y formulación claramente están orientadas a obtener respuestas punitivas ${ }^{\mathrm{v}}$. En los casos de abuso consciente o negligente de la investigación científica para legitimar determinadas opciones políticas, el mal, la investigación científica, es también en este caso la medicina. Solamente la existencia de un foro de discusión abierto y una comunidad crítica de investigadores que se adhiere a un riguroso y claro código ético tiene la capacidad de detectar y denunciar este tipo de prácticas.

\section{La criminología en España}

Los estudios empíricos sobre la delincuencia y las respuestas sociales a la misma es lo que se define como criminología. En el ámbito comparado la criminología es una disciplina y un área de estudios bastante consolidada. Existen titulaciones académicas reconocidas y tanto organizaciones públicas como privadas dedican presupuestos importantes a la realización de estudios criminológicos que permitan orientar o informar el diseño de políticas públicas. En El Reino Unido, por ejemplo, el Home Office dedica un presupuesto anual de 41 millones de libras a su departamento de estudios mientras que en los Estados Unidos el National Institute of Justice también cuenta con una financiación millonaria.

En España, por el contrario, la situación no es tan saludable, a pesar de unos buenos antecedentes históricos y el progreso que ha tenido lugar desde la transición democrática. En general la situación de la criminología en España está atrasada tal y como se manifiesta en diversos ámbitos.

a) No existen estudios homologados de criminología. A pesar de que España es uno de los países de la Unión Europea con un mayor número de alumnos y graduados de criminología, estos estudios no están homologados con los problemas que ello plantea en cuanto a control de calidad de la enseñanza impartida y posibilidades de desarrollo profesional. Aunque desde hace años se discuten propuestas para cambiar esta situación y el Consejo de Universidades aprobó un plan de licenciatura hace aproximadamente un año, la situación está paralizada y hay quien teme que la licenciatura no vaya a salir adelante. El plan no era desde luego el mejor plan que se podía aprobar y en opinión de algunos presentaba serios problemas (Medina, 2002), pero posiblemente era mejor que nada.

b) No existe una criminología administrativa en sentido estricto. En España no existen organismos similares al norteamericano National Institute of Justice dependiente del Departamento de Justicia en los Estados Unidos o al 
Research and Statistics Directorate dependiente del Home Office en Inglaterra y Gales que se encargue de realizar estudios criminológicos que informen la política criminal del gobierno. Existen notorias excepciones, particularmente en Cataluña, pero incluso en esta comunidad autónoma los medios son precarios si los comparamos a los existentes en el ámbito comparado y la influencia en el diseño de políticas no está claramente documento.

Ciertamente la Policia Nacional cuenta en nuestro país con un centro de estudios y también existe un gabinete estadístico en el Ministerio del Interior, pero sus actividades son fragmentarias, cuentan con pocos medios personales y materiales y sus objetivos y misiones son bastante limitadas. No pueden, con justicia, compararse a sus homólogos en los países con una criminología administrativa en sentido propio. Quizás la excepción más sobresaliente de ámbito nacional la representa el Consejo General del Poder Judicial que durante los últimos años ha apostado por la investigación sociolegal y criminólogica como un instrumento a la hora de resolver los problemas que son su competencia. Digna de elogio también ha sido la tendencia del CGPJ a colaborar con la comunidad de investigadores españoles y de hacer transparente sus actividades de investigación como una forma de impulsar el debate democrático e informado en torno a la justicia.

c) Quienes se dedican a la investigación criminológica lo hacen contra viento y marea y, en numerosas ocasiones, a costa de perjudicar su ascenso y supervivencia en un sistema académico y de investigación no solamente excesivamente jerarquizado y formalista que limita trayectorias personales de tipo interdisciplinar (por ejemplo, el sociólogo que se hace psicólogo, o el jurista que se hace criminólogo) y colaboraciones de esta naturaleza, sino también dominado por criterios disciplinares que no reconocen el valor de la investigación criminológica. El hecho de que la criminología sea una enseñanza no reglada, y lo que es más importante, ni un área de conocimiento reconocida significa que no existen plazas docentes para quienes deciden identificarse como criminólogos con todos los problemas que ello implica para quienes se dedican a la investigación criminológica que siempre son evaluados en cuanto que psicólogos, juristas o sociólogos sin que su aportación al saber criminológico sea seriamente tenida en cuenta.

Existen razones para la esperanza. A pesar de lo dicho existe un colectivo de investigadores formado por psicólogos, sociólogos, economistas, juristas, funcionarios de la administración pública y criminólogos que, con medios extremadamente precarios, se dedican de forma, si se quiere, un tanto quijotesca a la investigación criminológica en España. Este colectivo, la Sociedad Española de Investigación Criminólogica (SEIC), se constituyó formalmente en sociedad profesional en el año 2002 con el fin fundamental de fomentar la investigación criminólogica en nuestro país. La creación de este colectivo es sin duda alguna el desarrollo más importante que ha experimentado la criminología española durante la pasada década y, a pesar de las dificultades existentes, augura un futuro más prospero para nuestra disciplina que ya se ha manifestado en avances concretos tal y como la celebración de congresos científicos desde 1995, la creación de una 
página web, la colaboración en foros internacionales, el desarrollo de un código ético, la elaboración de un listado de investigadores, la propuesta de crear cursos de formación para políticos jóvenes, el nacimiento de esta revista, etc.

Como señalaba anteriormente existen razones para la esperanza. La creación de la SEIC ha servido para canalizar nuestras reivindicaciones y el volumen del grupo ha crecido considerablemente desde la primera reunión en 1995. En nuestro país junto a la comunidad de investigadores aunados en el REIC hay que contar también a otros investigadores que sin ser miembros de la sociedad estudian temas similares. En Oñati, por ejemplo, el Instituto Internacional de Sociología Jurídica, empleando criterios de excelencia, organiza eventos y supervisa estudiantes que escriben tesis sobre estos temas. La experiencia del Consejo General del Poder Judicial debería servir de modelo a otras instancias del sistema de justicia penal. Por otra parte, tanto el Instituto de la Mujer como el Instituto de la Juventud realizan y financian estudios que tienen un claro interés criminológico. Aunque desde la comunidad criminólogica somos conscientes que una colaboración más explícita con nosotros podría servir para mejorar la calidad de algunos de los estudios realizados desde estas instancias, no cabe duda que el esfuerzo realizado desde las mismas no puede ignorarse. $\mathrm{Ni}$ podemos ignorar que los Institutos de Criminología forman ahora parte cotidiana del mapa universitario español. Finalmente, la clase política es consciente de la necesidad de tratar la inseguridad ciudadana y es más que posible que en este contexto, como ha ocurrido previamente en otros países, se fomente de forma más activa la investigación criminólogica. Algunas formaciones políticas, de hecho, durante la pasada campaña electoral anunciaron, eso sí con menos fanfarria que otras medidas, la necesidad de fomentar el desarrollo de estudios criminológicos. Es triste, sin duda, que el futuro de nuestra disciplina vaya unido de forma pareja a la consolidación en la conciencia pública de un problema social.

\section{La Revista Española de Investigación Criminólogica}

En este contexto, los criminólogos que eventualmente fundamos la SEIC formalmente durante años reflexionamos sobre la necesidad de crear un foro de expresión en el que difundir los resultados de estudios criminológicos para facilitar el desarrollo de nuestra disciplina. Aunque quienes participamos en el proyecto criminológico no tenemos dificultades para publicar nuestras contribuciones, las mismas suelen ser publicadas en revistas que por su carácter disciplinar especializado no suelen llegar al conocimiento de criminólogos con una formación disciplinaria diferente. Ello evidentemente limita la posibilidad de un dialogo científico así como el conocimiento mutuo. Por esa razón, una de las primeras decisiones en la asamblea general del primer año en que formalmente funcionamos como sociedad fue la creación de una revista científica, la Revista Española de Investigación Criminológica.

La REIC quiere servir para elevar el nivel de las discusiones sobre el problema de la inseguridad ciudadana y la justicia penal española. Al mismo 
tiempo, en la SEIC somos conscientes de que los problemas que confrontamos los criminólogos españoles no son del todo únicos y que, de hecho, en el contexto latinoamericano la situación es en ocasiones más precaria si cabe. Nuestros colegas latinoamericanos se lamentan de la desaparición en dicho contexto de foros de expresión durante la última década en un período en el que debates sobre inseguridad ciudadana no han perdido vigencia y durante el cual la presión externa para imponer determinado tipo de soluciones a sus problemas político criminales no ha desaparecido. Por esa razón, y porque no abundan los foros criminológicos en castellano, la REIC nace con la pretensión de estrechar los vínculos de colaboración con los criminólogos hispanohablantes.

En otras secciones de esta página web se proporcionan los detalles sobre nuestra línea editorial, así como sobre las reglas de funcionamiento que rigen la revista, por lo que no abundaré demasiado en estos detalles. Señalar, al menos, que apostar por una publicación abierta de acceso gratuito y universal a través de la red no solo responde a consideraciones de tipo práctico (p.ej., costo económico), sino que es una decisión deliberada orientada a facilitar la difusión de nuestros estudios a la comunidad más amplia posible de forma que dicha comunidad se fortalezca y desarrolle una identidad más sólida. Indicar también que el empleo de un exhaustivo proceso de revisión tiene como cometido la difusión de buena práctica científica, así como elevar el tono científico y la calidad del debate. Y que esperamos que, por medio de la producción de un saber común sobre el fenómeno que se quiere regular y sobre las intervenciones que se emplean para regularlo, podamos extraer lecciones que sirvan para diseñar intervenciones más justas y eficaces.

Baste decir que la pelota ahora está encima de nuestro tejado. Finalmente tenemos el medio para expresar nuestra voz y para publicar nuestros estudios por el que tanto hemos luchado. El éxito de esta empresa en buena medida resta en nuestras espaldas. Desde la dirección de la REIC esperamos que el colectivo de criminólogos españoles y latinoamericanos que tienen acceso a la revista se anime a colaborar con este proyecto. El desarrollo de un sólido cuerpo de estudios sobre la realidad criminal y la justicia penal de nuestros países puede contribuir a sacarnos de la situación en la que ahora nos encontramos y a mejorar el modelo de formulación de políticas criminales en nuestro entorno. Y, a pesar de los obstáculos, todos somos más o menos conscientes de que poco a poco, sin duda más despacio de lo que nos gustaría, la situación de la criminología en España está mejorando.

Juanjo Medina, Editor Jefe de la REIC

iD https://orcid.org/0000-0003-4407-8830

\section{Referencias}

Beck, Ulrick. 1992. Risk Society. Towards a New Modernity. London: Sage. 
Campbell, Donald. 1988. The Experimenting Society. En E. S. Overman (Ed.). Methodology and Epistemology for Social Science: Selected Papers (pp. 290314). Chicago: University of Chicago Press.

Diez Ripolles, Jose Luis y Anabel Cerezo (Eds). Los problemas de la investigación empírica en criminología: La situación española. Valencia: Tirant lo Blanch.

European Opinión Research Group (EORG). 2003. Public Safety, Exposure to Drug-Related Problems and Crime. Bruselas: European Commission. Garland, David. 2001. The Culture of Control. Oxford: Oxford University Press. Hassemer, Winfried y Francisco Muñoz Conde. 1989. Introducción al Derecho Penal y la Criminología. Valencia: Tirant lo Blanch.

Hill, Michael. Ed. 1997. The Policy Process: A Reader. 2 Edición. Harlow: Prentice Hall.

Lopez Rey, Manuel. 1985. Compendio de criminología y política criminal. Madrid: Tecnos.

Medina, Juanjo. 2002. Reflexiones sobre la futura licenciatura en criminología. Revista Electrónica de Ciencia Penal y Criminología. http:/ criminet.ugr.es/recpc. Medina, Juanjo. En Prensa. Discursos políticos sobre inseguridad ciudadana en la historia reciente de España. Libro Homenaje a Alessandro Baratta.

National Audit Office. 2003. Getting the Evidence: Using Research in Policy Making. London: National Audit Office.

Parsons, Wayne. 1995. Public Policy. An Introduction to the Theory and Practice of Policy Analysis. Cheltenham: Edward Elgar.

Roberts, Julian and Loretta Stalans. 2000. Public Opinión, Crime, and Criminal Justice. Boulder, CO: Westview.

Sudman, Seymour y Norman Bradburn. 1982. Asking Questions. A Practical Guide to Questionnaire Design. San Francisco: Josey-Bass Publishers.

\footnotetext{
${ }^{\mathrm{i}}$ La traducción española de este informe será publicada en la REIC en breve.

${ }^{i i}$ Por ejemplo, se usa de forma indiscriminada y generalizada un índice de la delincuencia que pretende recoger todos los delitos registrados a pesar de lo inestable que el registro de determinados delitos es. Tiene mucho más sentido desglosar las categorías de delitos, centrarse en los más serios y presentar series temporales para aquellos delitos con una menor cifra oscura.

iii Sudman y Bradburn (1982, p.3) exponen un buen ejemplo sobre la Guerra de Corea de como pequeñas variaciones en la redacción de una pregunta resultan en diferentes respuestas:

Do you think the United Stated made a mistake in deciding to defend Korea, or not? (Gallup, Enero de 1951)

Mistake $\quad 49 \%$

Not a mistake $\quad 38 \%$

Don't know $\quad 13 \%$

Do you think the United States was right or wrong in sending American troops to stop the Communist invasion of South Korea? (National Opinion Research Center, Enero de 1951)

Wrong $\quad 36 \%$

Right $\quad 55 \%$

Don't know $\quad 9 \%$

En el ámbito político criminal, también sabemos que cuando se presentan preguntas genéricas los resultados son más punitivos que cuando se ofrecen preguntas más detalladas sobre diferentes alternativas.
} 
${ }^{\text {iv }}$ Resumiendo la literature en opinion pública y sanciones penales, Roberts y Stalans (2000, pp. 218-223) resaltan: "We could argue that the surveys of public attitudes that have been conducted are at best an oversimplification, and at worst misleading about the tenor of public views. The central deficiency of a public survey is that it usually poses a simple question which encourages (and indeed compels) people to respond in a simple way... a complex and emotional topic such as sentencing cannot be adequately researched in the same way that market researchers explore preferences for soft drinks. Before concluding that the public endorse harsher sentencing or that they oppose parole for inmates serving life terms, legislators and criminal justice officials should take a careful look at the research that has accumulated over the past forty years in several countries... Pollsters will doubtless continue to conduct, and the news media will continue to report, polls in which the public are simply asked whether sentences are too harsh or too lenient. And in all probability the public will continue to state that the courts are too lenient. In light of this, it is important that the results of more refined research be made available..."

v La alternativa metodologicamente correcta es: "A solution to this problem involves studying public opinion in a context in which respondents have a great deal more information at their disposal. This might be termed the case history approach, and has been followed by several researchers. The general strategy is to give people a specific case to read, this allowing them sufficient time to consider the many aspects of the case" (y que sentencia imponer)."The typical finding has been that when confronted with a specific case, the public are less punitive than when answering a general question" 